\title{
A study on recommender system considering diversity of items based on LDA
}

\section{Zhiying Zhang*}

Graduate School of Creative Science and Engineering,

Waseda University,

3-4-1 Okubo, Shinjuku-ku, Tokyo, 169-8555, Japan

Email: choshiei@akane.waseda.jp

*Corresponding author

\section{Taiju Hosaka}

Sansan, Inc.,

Aoyama Oval Building 13F,

5-52-2 Jingumae, Shibuya-ku, Tokyo, 150-0001, Japan

Email:wasewase@moegi.waseda.jp

\section{Haruka Yamashita}

School of Information and Computer Science,

Sophia University,

7-1, Kioi cho, Chiyoda-ku, Tokyo, 102-8854, Japan

Email: h-yamashita-1g8@sophia.ac.jp

\section{Masayuki Goto}

School of Creative Science and Engineering,

Waseda University,

3-4-1 Okubo, Shinjuku-ku, Tokyo, 169-8555, Japan

Email: masagoto@waseda.jp

\begin{abstract}
With the rapid development of information technology, a recommender system making use of users' behaviour data, such as browsing history or ratings for items, is now one of the important tools for searching contents or products. Recently, it has been shown that diversifying the recommendation lists in recommender systems could satisfy users' potential needs. In a previous research, the diversity of recommender system can be raised by the topic diversification method based on latent Dirichlet allocation (LDA); however, since the items belonging to the same topic are not diversified, the recommended items in the list shown to a user tend to be similar. Therefore, this research proposes a method for a recommendation system that diversifies items in each topic based on topic information obtained by LDA. Experimental results with MovieLens datasets demonstrate that our approach keeps accuracy of the recommendation and realises more diversified recommendation.
\end{abstract}

Keywords: recommender system; latent Dirichlet allocation; LDA; topic model; machine learning; diversity. 
Reference to this paper should be made as follows: Zhang, Z., Hosaka, T., Yamashita, H. and Goto, M. (2021) 'A study on recommender system considering diversity of items based on LDA', Asian J. Management Science and Applications, Vol. 6, No. 1, pp.17-31.

Biographical notes: Zhiying Zhang is a graduate student at the Waseda University, Japan. She received her Bachelor's degree from Shandong University in 2018. Her main research interests are machine learning and recommendation systems.

Taiju Hosaka works in Sansan, Inc., Japan. He received his Bachelor's degree from the Waseda University in 2018 and his Master's degree in 2020. His main research interests are machine learning and statistical modelling.

Haruka Yamashita is an Assistant Professor at the Department of Information and Communication Sciences, Sophia University, Japan. She received her PhD degree from the Keio University in 2015. Her research interests include machine learning, applied statistics, and multivariate data analysis.

Masayuki Goto is a Professor of School of Creative Science and Engineering, Waseda University, Japan. He received his DrE degree from the Waseda University in 2000. His research interests include data science, machine learning, business analytics, information theory and its application, and marketing analysis. He is a member of IEEE, INFORMS, the Institute of Electronics, Information and Communication Engineers, Japan Industrial Management Association, the Japan Society for Management Information, the Japanese Society for Artificial Intelligence, Business Model Association, and the Operations Research Society of Japan.

This paper is a revised and expanded version of a paper entitled 'A study on recommender system considering diversity of items based on LDA' presented at 2019 Asian Conference of Management Science \& Applications (ACMSA2019), Penglai, Shandong, China, 11-14 October 2019.

\section{Introduction}

In recent years, with the development of information technology, a huge amount of data on previous commodity purchase and the ratings for items have become possible to be accumulated in electric commerce (EC) sites. By analysing such data, recommendation systems are widely activated. So far, many recommendation systems have been proposed, which make use of previous commodity purchase data and taking each user's preference into consideration (Adomavicius and Tuzhilin, 2005; Umbrath and Hennig, 2009; Park et al., 2011; Lu et al., 2012; Bobadilla et al., 2013). Originally, Goldberg et al. (1992) designed the collaborative system for a group of users in order to make a list of emails that may interest them. Up to now, the recommendation system given by Breese et al. (1998) and Jin et al. (2010) are mainly applied to the field of marketing. Based on the information such as rating histories, purchase histories, or browsing log histories, recommendation systems pick out the items with a higher probability of user preference. While this approach may satisfy users' preferences, the items recommended tend to be similar to each other according to Xing et al. (2017), which mean similar items tend to be 
repeatedly recommended for each user, making the efficiency of recommendation declined.

In order to construct a good recommender system, McNee et al. (2006) shows that it is necessary to determine items for recommendation in consideration of not only purchase possibility but other measures to meet the users' satisfaction. One of such measures is 'diversity'. In recommendation based on the possibility of purchasing or preference, the accuracy of recommended items can be guaranteed. However, serendipity, with the meaning of unexpected and fortunate discoveries is one of the important factors for users to search a new item. Since the diversity of recommended items leads to serendipity to many users, the measure of diversity is often important to meet the users' satisfaction. According to Ziegler et al. (2005), recommendation that takes diversity into consideration may be possible to satisfy users' potential needs because it is possible to recommend items that they have not recognised so far.

Many researches proposed the recommendation algorithms considering diversity, and Ashkan et al. (2015) defined the method of constructing recommendation lists based on a Greedy method. In this method, the objective function to be learned consists of two terms:

1 the accuracy term obtained by recommending each item to the user

2 the diversity term calculated from the combination of items in the recommendation list.

The method sequentially adds items that can improve the objective function to the recommendation list. In addition, Xing et al. (2017) extracts latent topics behind items based on latent Dirichlet allocation (LDA) (Blei et al., 2003) and proposed an algorithm of constructing a recommendation list in which topics of items are diversified. Since each item can be represented by a distribution on the topics by applying LDA, it is possible to make use of the topic distribution for increasing diversity in the recommended item list. That is, if we construct an item list with the probabilities of the items belonging to each topic equally high, the diversity of the recommended item set should also be high. However, while this approach diversifies topics in the recommendation list from the viewpoint of the belonging probabilities to each topic; it does not guarantee the diversity of individual items. If we recommend an item with no unbiased topic distribution that means containing many topics, this way will also increase diversity in numbers. However, this method is undesirable from the viewpoint of item diversification and user satisfaction because characteristic items tend not to be included in the item set. The disadvantage is more pronounced when a user prefers multiple topics. For example, when recommending two movies to a user who likes both the genres of 'sci-fi' and 'horror' by the movie recommendation system, the following two types of recommendations are possible for the preferences of this user.

a Recommending two movies with both elements of 'sci-fi' and 'horror'.

b Recommending one movie with the element of 'sci-fi' and another movie with the element of 'horror'.

Although both (a) and (b) are recommendations satisfying multiple user preferences, (b) should be more desirable in terms of the diversity of individual items. A user who likes items in the genres of 'sci-fi' and 'horror' ought to prefer both an excellent item of 'sci-fi' and a good item of 'horror'. 
In this research, we propose an algorithm for constructing a recommendation list with restriction on similarity between items in the recommendation list by making use of a topic distribution of LDA. By using the information of topical distribution given by a trained LDA model, this strategy will increase not only the variety of topics of recommended items, but also the variety of individual items. That is, it is expected that the recommendation item list with higher diversity can be constructed under the same level of recommendation accuracy. In addition, we apply the proposed method to benchmark data and compare the proposed method with the conventional method from the viewpoints of both accuracy and diversity. By applying to the benchmark data, the effectiveness of the proposed method is clarified.

This paper is organised as follows. Section 2 introduces related works. Section 3 describes the algorithm of proposed method. Section 4 describes the experiments of proposed method, including experimental setup, evaluation metrics, results and considerations of the experimental results. Section 5 discusses the application and approaches of the proposed methods. Finally, Section 6 presents our conclusions and future issues.

\section{Related work}

Our work is related to the researches about LDA model (Blei et al., 2003) and also related to the researches about diversification in the recommendation systems (Xing et al., 2017). In the following parts, we will give a brief review of those works in this section.

\subsection{Latent Dirichlet allocation (Blei et al., 2003)}

LDA model is one of the representative topic models, which can be used to solve to various kinds of problems. It was originally proposed as a model for natural language processing, but recently, it is also applied to marketing analysis of the customers' purchase history data. In general, it is natural to assume that purchase history data are collection of behaviour histories of various users with completely different preferences, and the dataset consists of groups with different statistical properties. In topic models such as LDA, data with similar statistical characteristics tend to be put together into a group and each group with different statistical properties is represented as a topic. When LDA is applied to purchase history data, the distribution of the belonging probabilities to topics for each user and that of the appearance probabilities (i.e., purchasing probabilities) of items for each topic are assumed. In fact, Nishio et al. (2019) have proposed the method for finding the trigger item to grow users in terms of the purchase amounts based on the LDA. The various purchasing behaviours by users can be formulated by assuming latent variables taking values of topics, and the statistical relationship between users and items can be estimated by the purchasing history data.

Let user sets of $M$ people be $U=\left\{u_{1}, \cdots, u_{M}\right\}$, item sets of $N$ items be $V=\left\{v_{1}, \cdots\right.$, $\left.v_{N}\right\}$, topic sets of $K$ topics be $Z=\left\{z_{1}, \cdots, z_{K}\right\}$, respectively. Also, let $\theta_{m, k}$ denotes the probability that the user $u_{m}$ chooses the topic $z_{k}$ and $\boldsymbol{\theta}_{m}=\left(\theta_{m}, 1, \cdots, \theta_{m, K}\right)^{T}$ denotes the topical distribution of the user $u_{m}$. Similarly, let $\phi_{k, n}$ denotes the probability of that the item $v_{n}$ appears in the topic $z_{k}$, and let $\phi_{k}=\left(\phi_{k}, 1, \cdots, \phi_{k, N}\right)^{T}$ denotes the distribution of topic $z_{k}$ over the set of items. We employ symmetric Dirichlet priors $\operatorname{Dir}(\boldsymbol{\alpha})$ and $\operatorname{Dir}(\boldsymbol{\beta})$ 
with the hyper-parameters of $\boldsymbol{\alpha}=\left(\alpha_{1}, \cdots, \alpha_{K}\right)^{T}$ and $\boldsymbol{\beta}=\left(\beta_{1}, \cdots, \beta_{K}\right)^{T}$ on $\boldsymbol{\theta}_{m}$ and $\phi_{k}$ respectively. Then user preferences can be expressed by the probability $P\left(v_{n} \mid u_{m}\right)$ that user $u_{m}$ purchases the item $v_{n}$. It can be computed by equation (1).

$$
P\left(v_{n} \mid u_{m}\right)=\sum_{k=1}^{K} \iint \theta_{m, k} P\left(\theta_{m, k} \mid \alpha\right) \phi_{k, n} P\left(\phi_{k, n} \mid \beta\right) d \theta_{m, k} d \phi_{k, n} .
$$

By calculating equation (1), we can find the items that are likely to be preferred to each user respectively. Then we can decide the items that should be recommended to him/her.

Figure 1 This figure shows an example of how LDA works (see online version for colours)

Document of sports

With the NBA Finals all wrapped up, and everyone home from the bubble, it's

officially time to enter Offseason mode. Because of the coronavirus pandemic, the

normal timeline has been thrown off, and many events have been modified to meet

social distancing requirements, while others such as Las Vegas Summer League

have been canceled outright.
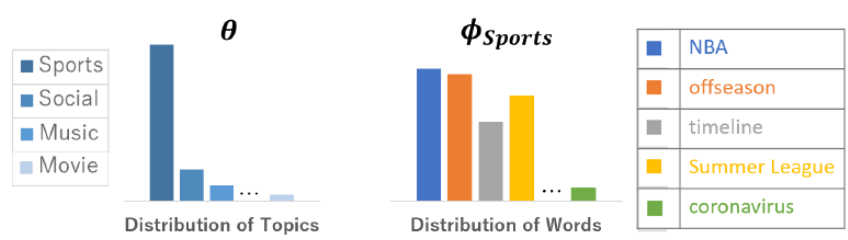

Notes: This article is an assay from CBS sports, so it has strong relation to the topic of sports. Meanwhile, there is a distribution that shows the appearance probability of words under the condition of each topic. While calculating equation (1), we can know that words with high value have more possibility to appear in this article.

\subsection{Research of diversity in recommendation (Xing et al., 2017)}

In the straight forward approach for the recommendation based on LDA, the recommender system constructs a list of recommended items for user $u_{m}$ by aggregating items with high estimated purchase probability for him/her. Here, in order to represent the item features more accurately, Xing et al. (2017) defined $\pi_{n, k}$ as the possibility that topic $z_{k}$ appears under the item $v_{n}$, and defined $\pi_{n}=\left(\pi_{n, 1}, \cdots, \pi_{n, K}\right)^{T}$ as the topic distribution of the item $v_{n}$. The recommendation based on LDA can be interpreted that items maximising $\boldsymbol{\theta}_{m}^{T} \boldsymbol{\pi}_{n}$ are sequentially added to the recommendation list. In this case, recommended items are determined basing on the user's favourite topics, and the algorithm tends to make a list with items that have similar topic distributions. Such a recommender system can show each user preferable items according to his/her favourite topics, which he/she has already recognised. However, if there are topics that each user may be fond of but have not yet been recognised, there is a possibility that more attractive item list can be recommended to them by diversifying the topics on the recommendation list. That is, in an actual recommendation system, it is often necessary to increase the diversity of the recommendation list as well as the suitability of items for each user's preference. 
To solve this problem, Xing et al. (2017) proposed a method to extend the recommendation list construction algorithm based on LDA, and to recommend items of various topics while keeping the relatively high accuracy of the recommendation. This research makes it easier to recommend items related to topics that have not been recommended yet.

It is implemented by an algorithm sequentially adding items to increase the diversification of a recommendation list. The algorithm reduces the utility of an adding item to a tentative recommendation list relative to the topics of items in the list. As a result, diversification of items in recommendation lists is realised in consideration of the user's preference. Let $L$ be the number of items recommended to each user, $I_{m}$ be the set of items not purchased by user $u_{m}$ and $S_{m}$ be the set of items recommended to user $u_{m}\left(S_{m}\right.$ $\left.\subseteq I_{m}\right)$. At this time, when item $v_{n}$ is added to the recommended item set $S_{m}$, a metric $f\left(v_{n} \mid\right.$ $S_{m}$ ) showing the quality of the recommendation is calculated by equation (2):

$$
f\left(v_{n} \mid S_{m}\right)=P\left(v_{n} \mid u_{m}\right)+\lambda \sum_{k=1}^{K} \theta_{m, k} \pi_{n, k} \prod_{v_{s} \in S_{m}}\left(1-\pi_{s, k}\right) .
$$

In this equation, the first term of the left hand side means the purchasing probability reflected by the preferences and the second term means diversity of topics. The parameter $\lambda$ determines the degree of diversification. Also, the construction algorithm of the recommendation list for $u_{m}$ is shown below.

\section{Algorithm:}

Step 1: $l=1$, set $S_{m}=\left\{\underset{v \in I_{m}}{\arg \max } P\left(v \mid v_{m}\right)\right\}$

Step 2: Calculate equation (3) is to find the item $v^{*}$ that maximises the quality of recommendation.

$$
v^{*}=\underset{v \in I_{m} \backslash S_{m}}{\arg \max } f\left(v \mid S_{m}\right) .
$$

Step 3: $l \leftarrow l+1$, set $S_{m} \leftarrow S_{m} \cup\left\{v^{*}\right\}$

Step 4: If $l<L$, go to step2. If $l=L$, end.

Note that this approach adds items one by one (i.e., the recommended item) as the optimal solution for each iteration. This approach is based on Greedy method (it will be described in detail in 2.3). Xing et al. (2017) also defined indicators of diversity. Diversity is calculated by equation (4).

$$
\text { diversity }=\frac{1}{M} \sum_{m=1}^{M}\left(1-\frac{\sum_{v_{i} \in S_{m}} \sum_{v_{j} \in S_{m} \backslash\left\{v_{i}\right\}} \operatorname{sim}\left(v_{i}, v_{j}\right)}{L(L-1)}\right) \text {, }
$$

In which $\operatorname{sim}\left(v_{i}, v_{j}\right)$ is defined as the cosine similarity between $\pi_{i}$ and $\pi_{j}$. The recommendation using the algorithm (Xing et al., 2017) is less accurate than the recommendation using original LDA (Blei et al., 2003) from the viewpoint of prediction accuracy for purchased items in future. In fact, the study of Xing et al. (2017) shows a trade-off relation between the accuracy and diversity through evaluation experiments. The diversity of the recommended item list can be increased by decreasing the accuracy of recommendation. In the actual application the attractive lists with high diversity should 
provide the new findings for users, because it provides high serendipity for users. The recommendation considering only the purchasing probability of each item makes the list consist of similar items and is not with high serendipity. Therefore, the diversity in recommended items lists as well as the users' preference for items would be both important to satisfy users in practice. The method of Xing et al. (2017) can be considered to be effective for this purpose.

\subsection{Greedy method (Edmonds, 1970)}

A greedy algorithm is a simple, intuitive algorithm that is used in the field of optimisation problems. The algorithm builds up a solution step by step, always choosing next step which is optimal with the intention of finding the overall optimal way to solve the entire problem. Although in many problems, a greedy algorithm does not always get an optimal answer, it can approximate an overall optimal solution.

This algorithm is used in the method of Xing et al. (2017) to build recommendation lists. They take every item that maximum the equation of utility, which is the local optimal solution into the recommendation list. It is logical under the circumstances that users only purchase items instead of the whole list, so we do not have the data of users' preferences for the lists. By using Greedy method, it is possible to make lists that help to decide the recommendation considering users' preference.

\section{Proposed method}

While the work of Xing et al. (2017) enables to recommend items with various topics, the dissimilarity between individual items is not guaranteed. In the case of recommending plural items belonging to many topics, the topics included in the recommendation list are diverse; however, a recommended item belonging to various topics may have high similarity with other items with the same various topics, as a result, diversity may lose from the user's point of view.

For example, if multiple items including both topic A and topic B are recommended to a user, these items recommended can be similar for him/her. On the other hand, if an item on topic A and another item on topic B are recommended, it is recognised from the viewpoint of the user that items on different topics are recommended to him/her, and thus the diversity of the recommended item list becomes high. Because of the above reason, it would be desirable to raise the diversity of items by recommending various items containing different topics respectively rather than recommend items that containing common multiple topic. That is, it seems to be better to recommend items with obvious characteristics, which means items belonging to relatively fewer common topics.

Therefore, in this research, we propose an algorithm for constructing more various recommendation lists considering the dissimilarity of each item. In the proposed method, an item with low similarity (high dissimilarity) to those included in the recommendation list is sequentially added to the list based on the same framework of the previous studies using a Greedy method. When item $v_{n}$ is added to the list of items $S_{m}$ for recommendation, the quality metric of the recommendation $g\left(v_{n} \mid S_{m}\right)$ is calculated by equation (5). Here, $\gamma$ is a parameter that determines the balance of degrees between user preference and diversification. Note that, when we use this algorithm for the actual 
recommendation, we have to decide the value of $\lambda$ in advance based on the experimental approach.

$$
g\left(v_{n} \mid S_{m}\right)=P\left(v_{n} \mid u_{m}\right)+\gamma \min _{v_{s} \in S_{m}}\left(1-\operatorname{sim}\left(v_{n}, v_{s}\right)\right) .
$$

This is the equation in which the topic diversity in equation (2) (Xing et al., 2017) is replaced by the item diversity in the list on the second term of the right-hand side. With this objective function, we take account of the item diversity in recommendation list while constructing algorithm. Since the similarity between items $\operatorname{sim}\left(v_{n}, v_{s}\right)$ is defined by the cosine similarity between the topic distributions, it is expected that we can make a recommendation list considering the item diversity as well. That is, the recommendation list constructed by the proposed method can diversify in terms of not only topics but also items. The specific algorithm of our proposal is shown as follows:

\footnotetext{
Algorithm:

Step 1: $l=1$, set $S_{m}=\left\{\underset{v \in I_{m}}{\arg \max } P\left(v \mid u_{m}\right)\right\}$
}

Step 2: Calculate equation (6) is to find the item $v^{*}$ that maximises the quality of recommendation.

$$
v^{*}=\underset{v \in I_{m} \backslash S_{m}}{\arg \max } g\left(v \mid S_{m}\right) .
$$

Step 3: $l \leftarrow l+1$, set $S_{m} \leftarrow S_{m} \cup\left\{0^{*}\right\}$

Step 4: If $1<L$, go to step 2. If $l=L$, output the list and end the algorithm

Figure 2 Here, we will use a numerical example to explain how these methods work (see online version for colours)

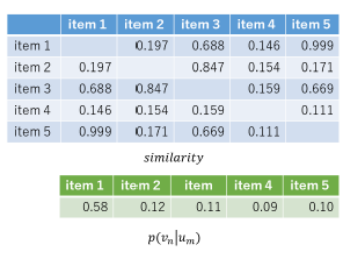

\begin{tabular}{|c|c|c|c|c|c|c|c|c|}
\hline & First item & \multicolumn{4}{|c|}{ Second item } & \multicolumn{3}{|c|}{ Third item } \\
\hline LDA only & item1 & \multicolumn{4}{|c|}{ item2 } & \multicolumn{3}{|c|}{ item3 } \\
\hline \multirow{3}{*}{ Xing et al. } & \multirow{3}{*}{ item1 } & \multicolumn{7}{|c|}{ After calculating $f\left(v_{n} \mid S_{m}\right)$, we got answers below $(\lambda=0.5)$ : } \\
\hline & & item2 & item3 & item 4 & item5 & item3 & item 4 & item5 \\
\hline & & 0.297 & 0.135 & 0.121 & 0.138 & 0.121 & 0.111 & 0.122 \\
\hline \multirow{3}{*}{$\begin{array}{l}\text { Proposed } \\
\text { Method }\end{array}$} & \multirow{3}{*}{ item1 } & \multicolumn{7}{|c|}{ After calculating $g\left(v_{n} \mid S_{m}\right)$, we got answers below $(\gamma=0.5)$ : } \\
\hline & & item2 & item3 & item 4 & item5 & item3 & item 4 & item5 \\
\hline & & 0.522 & 0.266 & 0.517 & 0.100 & 0.187 & 0.513 & 0.100 \\
\hline
\end{tabular}

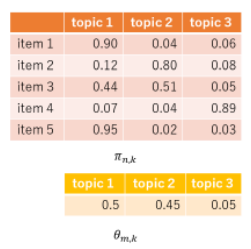

Notes: We suppose the number of items recommended to be 3 , and other assumptions can refer to the left figures. When using LDA, the items with higher $P\left(v_{n} \mid u_{m}\right)$ will be chosen, so in this example, item 1 , item 2 , and item 3 will be recommended. As for recommendation by Xing et al. (2017), they use $f\left(v_{n} \mid S_{m}\right)$ to measure the quality of the recommendation, so here, item 1, item 2 and item 5 will be recommended. However, while this method can recommend items with various topics, the dissimilarity between individual items is not guaranteed, such as item 1 and item 5 , we can see that the similarity between them is as high as 0.999 . To solve this problem, we change $f\left(v_{n} \mid S_{m}\right)$ to $g\left(v_{n} \mid S_{m}\right)$ in order to constructing more various recommendation lists considering the dissimilarity of each item, and in this example, item 1, item 2 and item 4 will be recommended. 


\section{Experiments}

In order to show the effectiveness of the proposed method, we perform an experiment using a benchmark data set.

\subsection{Experimental setup}

In this experiment, we use the publicly accessible dataset: the MovieLens data set. The number of users in the target data is $M=6,040$, the number of movies is $N=3,706$, and the total number of evaluations is $1,000,209$. The construction of the training data and test data and the evaluation of the model are based on the five-fold cross validation method. Here, the data are randomly divided into five groups equally for each user. Also, before each cross-validation learning, items less than the average rating value of each user in the training data are excluded from the training data and test data. As a result, it becomes an issue setting as to whether or not an item with a relatively high average rating value that may suit user's preference can be recommended to each user.

The number of the topics extracted by LDA is $K=10$, and the parameters of Dirichlet distribution are $\alpha_{k}=\frac{1}{K}(1 \leq k \leq K)$ and $\beta_{n}=\frac{1}{N}(1 \leq n \leq N)$. The learning of parameters was based on the variational Bayesian method according to Beal (2003). The effectiveness of the proposed method is examined in comparison with the recommendation by LDA only (equivalent to setting $\gamma=0$ in the proposed method) and the recommendation by the method of Xing et al. (2017).

As for the experimental environments, we describe as follows.

- $\quad$ Programming language: Python3.7.

- $\quad$ CPU: Intel(R) Core(TM)i7-8550U CPU @ 1.80GHz 1.99GHz.

- Running times: 25 minutes from training to test.

\subsection{Evaluation metrics}

In this study, we use two evaluation metrics by following the study of Xing et al. (2017). First, accuracy is used as an important evaluation metrics for the recommender system. Let $T_{m}$ be the set of items that the user $u_{m}$ is evaluating in the test data, and then the accuracy of the recommendation is calculated by the following equation:

$$
\text { precision }=\frac{1}{M} \sum_{m=1}^{M} \frac{\left|S_{m} \cap T_{m}\right|}{L},
$$

In addition, as a metrics to measure how diverse the recommended items can be, the diversity shown in equation (8) is also used as an evaluation index.

$$
\text { diversity }=\frac{1}{M} \sum_{m=1}^{M}\left(1-\frac{\sum_{v_{i} \in S_{m}} \sum_{v_{j} \in S_{m} \backslash\left\{v_{i}\right\}} \operatorname{sim}\left(v_{i}, v_{j}\right)}{L(L-1)}\right) \text {. }
$$

which is the same definition as equation (4). 


\subsection{Results}

The performances of the recommender systems by Xing et al. (2017) and our proposed method, the recommendation accuracy and diversity, depend on the setting of the parameters in equations (2) and (5). It is desirable to investigate the transition of the recommendation performance by setting the parameters.

Figure 3 shows the relationship between accuracy and diversity in each method when $L=10$. In the proposed method, experiments were performed using multiple parameters. We adjust parameter $\gamma$ of the proposed method in $1.0 \times 10^{-4} \leq \gamma \leq 1.0$ and adjust parameter $\lambda$ of Xing et al.'s (2017) method in $1.0 \times 10^{-3} \leq \lambda \leq 1.0$. The results are shown in Figure 3.

Figure 3 Relationship between accuracy and diversity (see online version for colours)

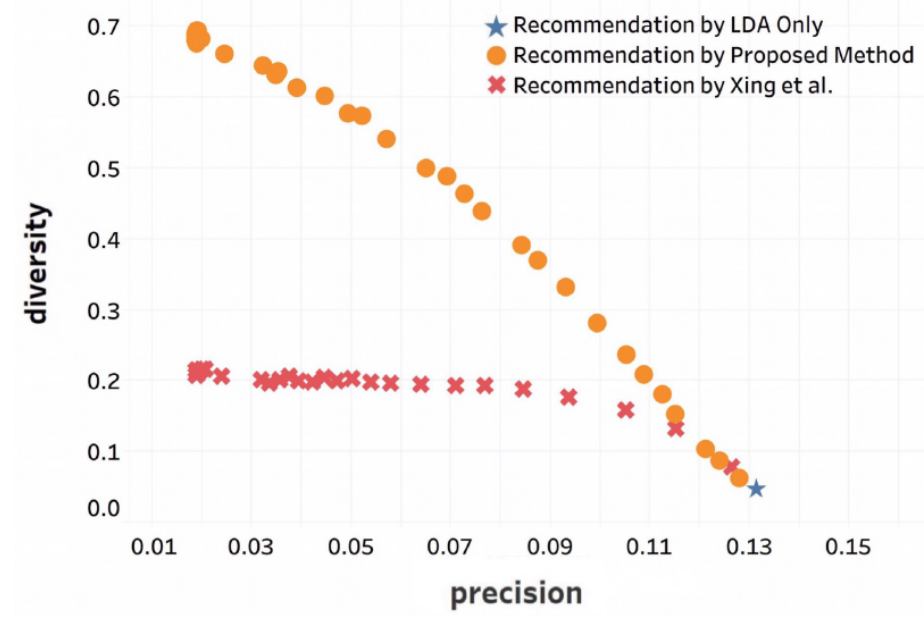

From Figure 3, it can be seen that the recommendation by the LDA shows the highest accuracy and lowest diversity. However, in the proposed method and the method of Xing et al. (2017) it becomes possible to obtain a recommendation list with diversity by changing the parameters. When the values of $\lambda$ and $\gamma$ are small, the diversification effect is also small, and the results are shown similar to the recommendation by LDA only. Conversely, as the values of these parameters increase, the accuracy of the recommendation decreases, and the diversity tends to increase. From this figure, it can be seen that comparing the results of the same accuracy, the proposed method exhibits higher diversity than the method of Xing et al. (2017). To the degradation of accuracy, the diversity of the proposed method increases greater than that of Xing et al. (2017), the graph shows that the performance of the proposed method is located in the upper right zone compared with the method of Xing et al. (2017) as a whole. In other words, the proposed method can be interpreted as being able to construct a more diverse item lists to each user while maintaining the same recommendation accuracy as the method of Xing et al. (2017).

Figure 4 shows the change in accuracy with respect to the number of recommended items $L$ in each method when $\gamma=0.0040$ and $\lambda=0.0004$, and Figure 5 shows the change in diversity. It can be seen from Figure 4 that when the number of recommended items $L$ 
increases, the accuracy is degraded in all methods. This is because the number of test data is constant, and the number of items evaluated by the user in the recommendation list has a ceiling. If the number of recommended items increases, it is considered that the accuracy will inevitably decrease. In particular, the accuracy of the proposed method decreases faster as the increase in the number of recommended items compared with other methods. This is because the proposed method attaches more importance to diversity in the recommendation list, and as the size of the list increases, different items of various topics come into the list, which may deviate from the user's preference.

In addition, it can be seen from Figure 5 that the variation of the diversity caused by the increase in the number of recommended items is small in the compared methods. On the other hand, it is confirmed that the diversity of the proposed method increases as the number of recommended items increases. The proposed method is an algorithm considering the diversity in the recommended item list, so when the size of the list becomes large, the diversity is also improved. In the case of a real situation designing a recommender system, the size of the list recommended to a user may not be large. Therefore, when the size of the recommended item list is relatively small, the performance of the proposed method is superior, reasonable and desirable in practice.

Figure 4 Relationship between the number of recommended items and precision (see online version for colours)

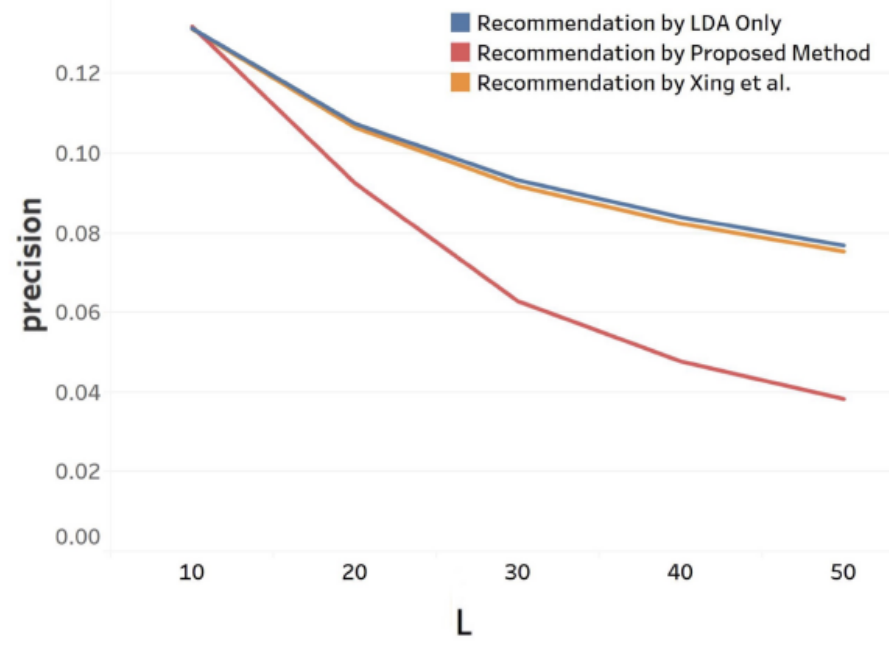

\subsection{Considerations of the experimental results}

From the results shown in Figures 3, 4, and 5, it can be said that the accuracy and diversity of the proposed method fluctuate greatly with the change of the number of recommended items. This is because the proposed method imposes constraints on the degree of similarity of individual items in the recommended item lists. If the number of recommended items increases, the combination of items will increase rapidly and it will become more difficult to recommend items that satisfy users' preference, thus the constraints will become much stronger. Hence, if it is desired to increase the number of recommended items without excessively increasing the diversity in the recommendation 
list, reducing the value of $\gamma$ and ease the restriction of the proposed method is necessary. Figures 4 and 5 are the results of the setting of fixed parameters in the objective functions. In order to adjust the parameters for a desirable recommender system, it is important to set the parameter in the objective function appropriately by adapting to the number of recommended items to each user.

Figure 5 Relationship between the number of recommended items and diversity (see online version for colours)

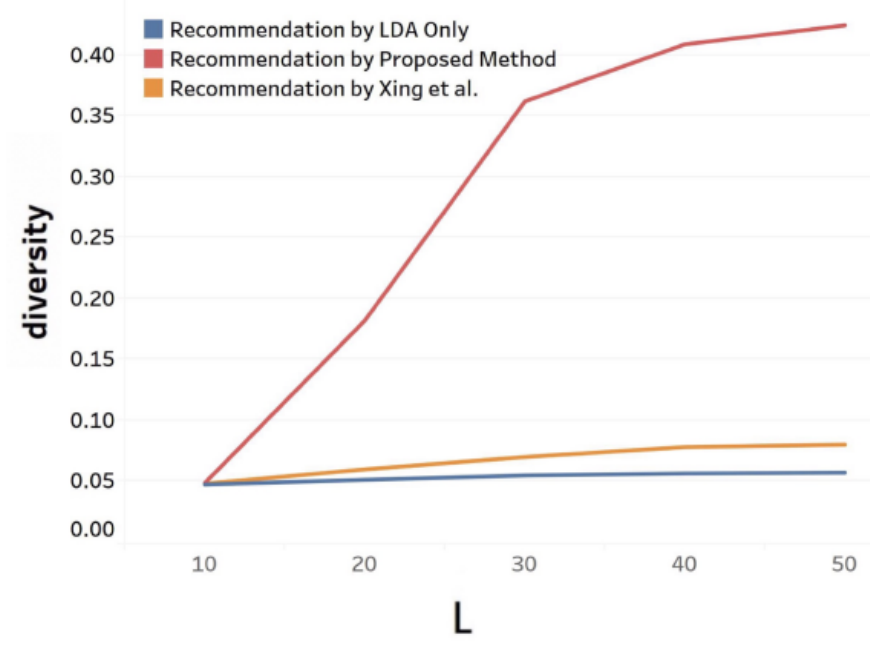

\section{Discussion}

\subsection{Application of the proposed methods in the actual marketing recommendation systems}

Although the experiment shows that the proposed method enables us to recommend various items considering the diversity of the recommendation, it has not evaluated how it makes the purchase probabilities or satisfaction increase. It is, therefore, necessary to incorporate the proposed method into an actual recommendation system and evaluate the effect quantitatively using systematic experiments such as $\mathrm{AB}$ test as a future task.

In addition, we used the MovieLens data with the ratings of movies for experimental evaluation of the proposed method in this paper. However, the actual application of the proposal method is mainly in retailer's online shops and the stores dealing with a variety of items. The diversity of the recommended items in the actual marketing should be more important compared to the MovieLens data in the experiment. Under the circumstance that the diversity of the recommended items is important, our method should be preferable to the actual application.

Also, we have to consider the cold start problem according to Schein et al. (2002) to realise the actual recommendation system based on the proposal. Since items are sold in a dynamic way and new users join in actual systems constantly and our proposed method requires a large amount of data about customers and items to make accurate recommendations, it may not work well for new items or new users added to the dataset. 
Several researches showed that the recommendation approach would not consider items that no one has evaluated yet (Rashid et al., 2008; Crane, 2011). In this case, the complementary approach for the new items/users should be required. For example, in the case of movies, by analysing the text information of a movie with a method such as Doc2vec according to Le and Mikolov (2014), it is possible to accurately estimate the similarity of a new movie to the ones that have already been evaluated. Also, the geographical data of the users can be obtained, so the similarities of the users are available.

\subsection{Approaches of the proposed method}

Here, let's consider the approaches we applied in our proposal: the estimation of the purchase probabilities of items for each user, and the optimisation of the items to be recommended [i.e., equation (3)] based on the Greedy algorithm (Edmonds, 1970).

In this study, we applied LDA model for the estimation of the users' purchasing probability for each item. For the estimation of the purchasing ratio, some alternative approaches are possible to be applied if the model can estimate latent topics of each item. For example, the probabilistic latent semantic analysis model (PLSA model) proposed by Hofmann (1999) is a fundamental topic model and can be applied to the purchase history data. The PLSA model assumes latent topics are behind the co-occurrence data of users and items, so the topic distributions of each existing item can be estimated. Note that there are papers such as Hyung and Lee (2013) and Jin et al. (2010) that extended the structure of PLSA for the recommendation systems. However, these latent class models are not generative models and the parameter estimation is based on likelihood principle. Since LDA model is based on Bayesian modelling, we can expect relatively high estimation accuracy for statistical learning. Therefore, LDA model is reasonable for the application to recommender system with respect to item diversity.

On the other hand, the optimisation for recommendation should be considered. In our approach based on Greedy method, it is not guaranteed to find the global solution although it is easy to implement. Therefore, other heuristics-based approaches such as iterative improvement according to Dorn (1995) and evolutionary computation according to Silva et al. (2010) can be considered. It is also a future task to evaluate whether the heuristic searching algorithms can be effective for this problem from the viewpoints of both recommendation accuracy and computational complexity.

\section{Conclusions and future issues}

In this research, we proposed a constructive algorithm for a recommendation list to make recommendations with high diversity while maintaining relatively high accuracy by considering the dissimilarity between items in the list. The proposed algorithm is based on the method by Xing et al. (2017) making use of a topic model and takes account of the item similarity measured by the topic distributions. Then, we applied the proposed method to benchmark data and showed its effectiveness from the viewpoints of both recommendation accuracy and the item diversity. In the evaluation experiment using MovieLens data set, compared with the conventional methods, the proposed method shows higher diversity with the same degree of accuracy. That is, it is possible to improve the diversity in the recommendation list while keeping the accuracy of 
recommendation and to anticipate further improvement of the user satisfaction by using the proposed method.

However, some limitations still remain in this study. In the experiment it is confirmed that the proposed method fluctuates greatly in accuracy and diversity with the changes in the number of recommended items. It is, therefore, necessary to adjust the parameters appropriately according to the number of recommended items when using the proposed method. In the future, it will be an issue to conduct an evaluation of the cost for adjusting parameters. Moreover, it is necessary to adjust the proposed model in the actual recommendation continuously because we should clarify how the proposed method improves customer satisfaction and purchasing probability. Although the proposed method considers both the recommendation accuracy and the item diversity, we cannot evaluate the customer satisfaction and the sales impact without actual application.

\section{Acknowledgements}

The authors thank Mr. Tianxiang Yang and Mt. Gendo Kumoi of Waseda University for their supportive discussions for this research.

\section{References}

Adomavicius, G. and Tuzhilin, A. (2005) 'Toward the next generation of recommender systems: a survey of the state-of-the-art and possible extensions', IEEE Transactions on Knowledge and Data Engineering, Vol.17, No. 6, pp.734-749.

Ashkan, A., Kveton, B., Berkovsky, S. and Wen, Z. (2015) 'Optimal greedy diversity for recommendation', Proceedings of the Twenty-Fourth International Joint Conference on Artificial Inteligence, pp.1742-1748.

Beal, M.J. (2003) Variational Algorithms for Approximate Bayesian Inference, $\mathrm{PhD}$ thesis, Gatsby Unit, University College London.

Bobadilla, J., Ortega, F., Hernando, A. and Gutierrez, A. (2013) 'Recommender systems survey', Knowledge-Based Systems, Vol. 46, pp.109-132.

Breese, J.S., Heckerman, D. and Kadie, C. (1998) 'Empirical analysis of predictive algorithms for collaborative filtering', Uncertainty in Artificial Intelligence, Vol. 14, pp.43-52.

Blei, D.M., Ng, A.Y. and Jordan, M.I. (2003) 'Latent Dirichlet allocation', Journal of Machine Learning Research, Vol. 3, pp.993-1022.

Crane, M. (2011) The New User Problem in Collaborative Filtering, Thesis for the degree of Master of Science, Department of Computer Science, University of Otago, Dunedin, New Zealand.

Dorn, J. (1995) 'Iterative improvement methods for knowledge-based scheduling', AI Communications, Vol. 8, No. 1, pp.20-34.

Edmonds, J. (1970) 'Submodular functions, matroids, and certain polyhedra', International Conference on Combinatorial Structures and their Applications, pp.69-87.

Goldberg, D., Nichols, D., Oki, B.M. and Terry, D. (1992) 'Using collaborative filtering to weave and information tapestry', Communications of the ACM, Vol. 35, No. 12, pp.61-70.

Hofmann, T. (1999) 'Probabilistic latent semantic analysis', Proc. of UAI' 99.

Hyung, Z. and Lee, K. (2013), 'Recommending music based on probabilistic latent semantic analysis on Korean radio episodes', 2013 Ninth International Conference on Intelligent Information Hiding and Multimedia Signal Processing, pp.472-476. 
Jin, B.W., Cho, Y.S., and Ryu, K.H. (2010) 'Personalized e-commerce recommendation system using RFM method and association rules', Journal of the Korea Society of Computer and Information, Vol. 15, No. 12, pp.227-235.

Le, Q. and Mikolov, T. (2014) 'Distributed representations of sentences and documents', Proceedings of Thirty-First International Conference on Machine Learning, pp.1188-1196.

Lu, L., Medo, M., Yeung, C.H., Zhang, Y., Zhang, Z. and Zhou, T. (2012) 'Recommender systems', Physics Reports, Vol. 519, No. 1, pp.1-49.

McNee, S.M., Riedl, J. and Konstan, J.A. (2006) 'Being accurate is not enough: how accuracy metrics have hurt recommender systems', Proceedings of International Conference on Human Factors in Computing Systems, pp.1097-1101.

Nishio, Y., Ito, H., Yamashita, H. and Goto, M. (2019) 'A new analytical model for customer growth considering potential purchasing preferences', Total Quality Science, Vol. 4, No. 3, pp.148-159.

Park, D.H., Kim, H.K., Choi, I.Y. and Kim, J.K. (2011) 'A literature review and classification of recommender system research', Expert Systems with Applications, Vol. 39, No. 11, pp.10059-10072.

Rashid, A.M., Karypis, G. and Riedl, J. (2008) 'Learning preferences of new users in recommender systems: an information theoretic approach', ACM SIGKDD Explorations Newsletter, Vol. 10, pp.90-100.

Schein, A.I., Popescul, A., Ungar, L.H. and Pennock, D.M. (2002) 'Methods and metrics for cold-start recommendations', Proceedings of the 25th Annual International ACM SIGIR Conference on Research and Development in Information Retrieval, pp.253-260.

Silva, N.B., Tsang, R., Cavalcanti, G.D. and Tsang, J. (2010) 'A graph-based friend recommendation system using genetic algorithm', IEEE Congress on Evolutionary Computation, pp.1-7.

Umbrath, A.S.R.W.W. and Hennig, L. (2009) 'A hybrid PLSA approach for warmer cold start in folksonomy recommendation', Recommender System and the Social Web, pp.10-13.

Xing, X., Sha, C. and Niu, J. (2017) 'Improving topic diversity in recommendation lists: marginally or proportionally', Proceedings of APWeb-WAIM 2017, pp.142-150.

Ziegler, C.N., McNee, S.M., Konstan, J.A. and Lausen, G. (2005) 'Improving recommendation lists through topic diversification', Proceedings of Association for Computing Machinery on World Wide Web, pp.22-32. 